\title{
The Radioprotective Effect of Procaine and Procaine-Derived Product Gerovital H3 in Lymphocytes from Young and Aged Individuals
}

\author{
Anca Ungurianu $\left(\mathbb{D},{ }^{1}\right.$ Denisa Margina $\left(\mathbb{D},{ }^{1}\right.$ Claudia Borsa $\mathbb{D}^{2},{ }^{2}$ Cristina Ionescu, ${ }^{2}$ \\ Gudrun von Scheven, ${ }^{3}$ Lucie Oziol, ${ }^{4}$ Philippe Faure $\mathbb{D}^{5},{ }^{5}$ Yves Artur, ${ }^{5}$ Alexander Bürkle, ${ }^{3}$ \\ Daniela Gradinaru $\mathbb{D},{ }^{1}$ and Maria Moreno-Villanueva $\mathbb{D D}^{3,6}$ \\ ${ }^{1}$ Department of Biochemistry, Faculty of Pharmacy, Carol Davila University of Medicine and Pharmacy, \\ RO-020956 Bucharest, Romania \\ ${ }^{2}$ Department of Biology of Aging, Ana Aslan National Institute of Gerontology and Geriatrics, RO-011241 Bucharest, Romania \\ ${ }^{3}$ Department of Biology, Molecular Toxicology Group, University of Konstanz, D-78457 Konstanz, Germany \\ ${ }^{4}$ Faculty of Pharmacy, CNRS UMR 8079, University of Paris-Sud, F-92296 Châtenay-Malabry, France \\ ${ }^{5}$ Centre for Taste and Feeding Behavior, UMR CNRS 6265-INRA 1324-University of Burgundy-AgroSup, F-21000 Dijon, France \\ ${ }^{6}$ Department of Sport Science, Human Performance Research Centre, University of Konstanz, D-78457 Konstanz, Germany
}

Correspondence should be addressed to Daniela Gradinaru; daniela.gradinaru@umfcd.ro

and Maria Moreno-Villanueva; maria.moreno-villanueva@uni-konstanz.de

Received 2 February 2020; Revised 5 May 2020; Accepted 8 May 2020; Published 25 June 2020

Academic Editor: Ji C. Bihl

Copyright (c) 2020 Anca Ungurianu et al. This is an open access article distributed under the Creative Commons Attribution License, which permits unrestricted use, distribution, and reproduction in any medium, provided the original work is properly cited.

\begin{abstract}
Ionizing radiation induces genomic instability in living organisms, and several studies reported an ageing-dependent radiosensitivity. Chemical compounds, such as scavengers, radioprotectors, and modifiers, contribute to reducing the radiationassociated toxicity. These compounds are often antioxidants, and therefore, in order to be effective, they must be present before or during exposure to radiation. However, not all antioxidants provide radioprotection. In this study, we investigated the effects of procaine and of a procaine-based product Gerovital H3 (GH3) on the formation of endogenous and X-ray-induced DNA strand breaks in peripheral blood mononuclear cells (PBMCs) isolated from young and elderly individuals. Interestingly, GH3 showed the strongest radioprotective effects in PBMCs from young subjects, while procaine reduced the endogenous amount of DNA strand breaks more pronounced in aged individuals. Both procaine and GH3 inhibited lipid peroxidation, but procaine was more effective in inhibiting mitochondria free radicals' generation, while GH3 showed a higher antioxidant action on macrophage-induced low-density lipoprotein oxidation. Our findings provide new insights into the mechanisms underlying the distinct effects of procaine and GH3 on DNA damage.
\end{abstract}

\section{Introduction}

Irradiation with ionizing radiation (IR) can induce mutations, cancer, and ageing in living organisms [1]. IR leads to oxidizing events causing cellular damage through direct interactions targeting macromolecules or via the free radicals produced by the radiolysis of water. These oxidizing events are amplified by endogenous cellular reactions, which further induce oxidative damage to DNA, lipids, proteins, and other molecules [2]. The basic mechanisms of radiationinduced lipid peroxidation have been previously summarized [3]. It is well known that reactive electrophilic compounds are formed during lipid peroxidation (mainly alpha and beta-unsaturated aldehydes) and can further alter DNA exhibiting both genotoxic and mutagenic actions; among them, 4-hydroxynonenal (4-HNE) is known for its 
genotoxic effects, while malondialdehyde (MDA) for its mutagenic ones [4]. Moreover, aldehyde-derived lipid peroxidation products can induce DNA strand breaks via oxidation of double bonds [5].

Immune cells are among the most radiosensitive cells in the body. However, their response to radiation depends on radiation type, dose, and dose rate. Both immunosuppressive and immune activating consequences were observed after high IR doses, while the effects of low doses are still controversial [6]. In fact, significant interindividual variability of radiation-related oxidative status [7] and DNA damage [8, 9] has been reported in human lymphocytes. Several cellular processes, such as defence against oxidative stress and DNA repair or telomere shortening and inflammatory pathways, may contribute to the relationship between aging and radiosensitivity [10]. Radiation contributes to the generation of reactive oxygen species (ROS), resulting in increasing amounts of cellular damage and aging. The link between radiosensitivity and aging was investigated in both healthy individuals and cancer patients, finding that the number of radiosensitive individuals increased with age in both groups [11].

Generally, antioxidants are considered to be of great interest for both radioprotection strategies [12-15] and antiageing therapies [16-18]. Radioprotective molecules may prevent the formation and facilitate the removal of free radicals, reinforce natural antioxidant systems, enhance DNA repair, reduce the postradiation inflammatory response, and even delay cellular division allowing more time for cells to undergo restorative processes or initiate apoptosis [15]. Furthermore, radioprotectors must exhibit radical-scavenging properties and antioxidant activity; however, not all antioxidants provide radioprotection [19].

Procaine was synthesized by Alfred Einhorn in 1905 and introduced in clinical practice as Novocain, becoming a local anaesthetic prototype. Procaine binds to membrane constituents and modulates a series of ion channels, interacts with membrane phospholipids, and induces changes in membrane fluidity depending on its concentration [20, 21]. Also, mitochondria, which are considered the powerhouses of the cell, are a potential target for general and local anaesthetics [22]. Procaine and its metabolites affect several biochemical and cellular processes like membrane conductance [20], oxidative phosphorylation [23], mitochondrial function and structure [24], monoamine oxidase activity [25], and DNA methylation [26]. Although Gerovital H3 (GH3) is a procaine-based preparation, its effectiveness has been disputed $[27,28]$. Nevertheless, the antioxidant actions of procaine and $\mathrm{GH} 3$ were reported in several in vitro studies [29-31]. Also, it was reported that high concentrations $(20 \mathrm{mM})$ of procaine inhibit DNA repair in bacteria [32].

In this context, we hypothesized that procaine might be a radioprotector, and we set out to investigate the effects of procaine and GH3 on the formation of endogenous and $\mathrm{X}$-ray-induced DNA strand breaks in immune cells isolated from young versus aged individuals. Furthermore, we used specific and sensitive in vitro assessments to determine their efficiency in preventing lipid peroxidation of various biological samples (lymphoblastoid cells, mitochondria, human serum, and oxidized LDL).
To the best of our knowledge, there are no prior studies reporting on the effect of low doses of procaine on radiation-induced DNA damage in human cells.

\section{Materials and Methods}

2.1. Chemicals, Drugs, and Reagents. Procaine hydrochloride (CAS No. 51-05-8) and all other routine reagents were of the highest purity commercially available and were purchased from Sigma-Aldrich (St. Louis, MO, USA). Commercially available Gerovital H3 (GH3) (Zentiva, Romania-approved by the National Agency of Medicines and Medical Devices according to no. 1583/2012/01 governmental order) injectable solution ( $2 \%$ procaine hydrochloride, $0.12 \%$ benzoic acid, $0.10 \%$ potassium metabisulphite, $0.01 \%$ disodium phosphate, and $\mathrm{pH} 3.3$ ) was purchased from a national pharmacy. To test comparatively the effect of $\mathrm{GH} 3$ versus the procaine effect, a working solution of $2 \%(w / v$, in distilled water) procaine hydrochloride was prepared and systematically used in each experimental model.

2.2. Assessment of DNA Strand Breaks. Peripheral blood mononuclear cells (PBMCs) were obtained from the whole blood by Biocoll (Biochrome AG, Germany) densitygradient centrifugation. The venous blood was drawn from volunteers using S-Monovettes (Sarstedt, Germany). Subjects were non-smokers and healthy female or male volunteers between 24 and 77 years of age. Ethical approval was obtained from the Ethics Committee of University of Konstanz. A signed Informed Consent was obtained from each subject. Non-stimulated PBMCs $\left(2 \times 10^{6}\right.$ cells $\left./ \mathrm{mL}\right)$ from two individual groups, elderly (71 \pm 6 years, $n=12$ individuals) and young ( $27 \pm 3$ years, $n=12$ individuals) subjects, were treated with $0,0.25,0.5$, or $1 \mathrm{mM}$ procaine or $\mathrm{GH} 3$ (in procaine hydrochloride equivalents) and incubated for 24 hours at $37^{\circ} \mathrm{C}$ in $5 \% \mathrm{CO}_{2}$-humidified atmosphere. Thereafter, cell suspension was removed by centrifugation, and PBMCs were suspended in isotonic buffer $(0.25 \mathrm{M}$ mesoinositol, Sigma-Aldrich, CAS No. 87-89-8; $10 \mathrm{mM}$ sodium phosphate, pH 7.4, Sigma-Aldrich, CAS No. 7601-54-9; and $1 \mathrm{mM}$ magnesium chloride, Sigma-Aldrich, CAS No. 7786-30-3) and irradiated on ice with $2,4,6$, or $8 \mathrm{~Gy}$ ionizing radiation $(70 \mathrm{kV}, 30 \mathrm{~mA}, 70 \mathrm{~cm}$ distance, $1.25 \mathrm{~mm}$ Al filter; Biological $\mathrm{X}$-ray Irradiator X-RAD $225 \mathrm{iX}$ from Precision X-ray, Inc., North Branford, USA). After irradiation, the cells were processed by a liquid handling device to assess DNA strand breaks. DNA strand breaks were quantified using the automated fluorescence-detected alkaline DNA unwinding (FADU) assay $[33,34]$. Due to the automation of the technical process, the automated FADU assay accomplishes a high reproducibility and sensitivity and has been successfully applied in several studies [35-47]. This method is based on the progressive DNA unwinding (denaturation) under controlled alkaline $\mathrm{pH}$, time, and temperature conditions $[33,34]$. All the automated analytical steps were performed using the TECAN Genesis RSP 100-LHD equipment (TECAN AG, Hombrechtikon, Switzerland). The dye SYBR ${ }^{\circledR}$ Green (Invitrogen, Darmstadt, Germany) that specifically binds to the double-stranded DNA (nonunwound) was used. 
A decrease in the fluorescence intensity of $\mathrm{SYBR}^{\circledR}$ Green was measured (492 nm excitation and $520 \mathrm{~nm}$ emission wavelengths) indicating an increase of DNA unwinding and, hence, a rise in the number of DNA breaks. Unwinding is expressed in Gy dose equivalent as described by [48]. The effects of procaine and $\mathrm{GH} 3$ treatment on endogenous DNA stand breaks in nonirradiated PBMCs were also evaluated using different concentrations. Previous to the radioprotection experiments, PBMCs obtained from few young subjects were incubated with 2, 3, 5, and $10 \mathrm{mM}$ of either procaine or GH3. Concentrations of procaine and GH3 of $3 \mathrm{mM}$ and higher showed a genotoxic effect as measured by DNA strand breaks formation, while $2 \mathrm{mM}$ of procaine did not have any protective effect or even induced DNA strand breaks (Figure S1). Therefore, $1 \mathrm{mM}$ and lower concentrations of both compounds were considered for further experiments.

2.3. Lipid Peroxidation in Jurkat Cell Membranes. Jurkat lymphocytes (T lymphoblasts, European Collection of Cell Cultures, UK) cultured in suspension in the RPMI 1640 medium (Sigma-Aldrich, Product No. SLM-240-B) supplemented with $10 \%$ FBS $\left(5 \times 10^{5}\right.$ cells/well $)$ were incubated for 4 hours at $37^{\circ} \mathrm{C}$ in $5 \% \mathrm{CO}_{2}$-humidified atmosphere, with different concentrations of procaine or GH3: 0 (control), 2.5, 5.0 , and $10 \mathrm{mM}$ procaine hydrochloride equivalents. Lipid peroxidation was induced by cumene hydroperoxide (CuOOH, Sigma-Aldrich, CAS No. 80-15-9). Cells treated with curcumin (Sigma-Aldrich, CAS No. 458-37-7) (0, 2.5, 5 , and $10 \mathrm{mM}$, in DMSO), a known antioxidant molecule, were used as a positive control. The lipid peroxidation of the cell membrane was further monitored with the fluorescent probe DPPP (diphenyl-1-pyrenylphosphine, Thermo Fisher Scientific/Invitrogen/Molecular Probes ${ }^{\mathrm{TM}}$, Eugene, Oregon, USA) according to the procedure described by Margina et al. [49, 50]. Cell suspensions were labelled with $5 \mu \mathrm{M}$ DPPP for 20 minutes in the dark, at room temperature, and were further treated with $10 \mu \mathrm{M} \mathrm{CuOOH}$ to induce lipid peroxidation. Fluorescence emission spectra between $360 \mathrm{~nm}$ and $410 \mathrm{~nm}$ (excitation set at $351 \mathrm{~nm}$ ) were recorded every 2 minutes, for 18 minutes using a Perkin-Elmer LS 50B spectrofluorometer, equipped with an externally thermostatic cell holder. Maximum fluorescence intensity was measured for an emission wavelength of $380 \mathrm{~nm}$ as previously described. Susceptibility to lipid peroxidation of treated samples and control cells were expressed as relative fluorescence units (RFU) after time-dependent exposure to $\mathrm{CuOOH}$.

2.4. Lipid Peroxidation in Rat Liver Mitochondria. Amplex Red (N-acetyl-3,7-dihydroxyphenoxazine, Life Technologies, Grand Island, NY, USA) proved to be a versatile probe which can be applied for the assessment of fatty acid hydroperoxides, $\mathrm{H}_{2} \mathrm{O}_{2}$, and other ROS in isolated mitochondria [51-53]. Crude liver mitochondrial fraction was obtained from a 3-month-old Wistar rat by differential centrifugation, using the protocol described by Wieckowski et al. [54]. The study was approved by the local Animal Ethics Committee at Carol Davila University of Medicine and Pharmacy, and all procedures were carried out according to the Directive $86 / 609$ EEC guidelines for the care and use of laboratory animals. Mitochondrial preparations (standardized for a protein content of $0.3 \mathrm{mg}$ protein $/ \mathrm{mL}$ phosphate buffer $20 \mathrm{mM}$, $\mathrm{pH} 7.3)$ were incubated for 10 minutes at room temperature $\left(25^{\circ} \mathrm{C}\right)$ in a $1: 1$ ratio with sodium succinate $(0.05 \mathrm{M}$, SigmaAldrich, CAS No. 150-90-3). Different procaine and GH3 volumes were added to reaction mixture, corresponding to 0 (control), $0.5,1.0,2.0,5.0$, and $10 \mathrm{mM}$ procaine hydrochloride final concentrations in the samples. After the addition of Amplex Red working solution $(75 \mu \mathrm{M})$, the samples were incubated for 10 minutes at room temperature and protected from light. The mixture was diluted to $2000 \mu \mathrm{L}$ with PBS 1x before reading at $585 \mathrm{~nm}$ emission wavelength after excitation to $544 \mathrm{~nm}$ on a LS 50B Fluorescence Spectrometer from Perkin-Elmer. The measurements were conducted versus a control mitochondrial sample without procaine or GH3. Also, for each experiment, a blank was prepared, containing Amplex Red working solution plus PBS 1x, in order to assess the probe's autofluorescence. Results were expressed as lipid peroxidation inhibition in percentage (\%), calculated from the recorded fluorescence as relative fluorescence units (RFU), using the following equation: $\left[\left(\mathrm{RFU}_{\text {Control }}-\mathrm{RFU}_{\text {Samples }}\right) / \mathrm{RFU}_{\text {Control }}\right] \times 100$.

2.5. Lipid Peroxidation in Human Serum. Serum samples were obtained from four healthy adult volunteers-students from the Faculty of Pharmacy, Carol Davila University of Medicine and Pharmacy. Ethical approval for collecting the peripheral venous blood from human subjects was obtained from the Carol Davila University of Medicine and Pharmacy Ethics Committee. Serum lipoprotein concentrates were isolated from each serum sample following centrifugation at $7000 \times \mathrm{g}, 4^{\circ} \mathrm{C}, 60 \mathrm{~min}$, on Amicon Ultra-10 k Centrifugal Filter Units 10.000 NMWL (Millipore). The assessment of lipid hydroperoxides in serum lipoprotein concentrates using the Amplex Red fluorescent probe was performed according to the method previously described $[52,53,55]$.

Briefly, $50 \mu \mathrm{L}$ of serum concentrate samples (standardized for a protein content of $0.5 \mathrm{mg}$ protein $/ \mathrm{mL}$ phosphate buffer $20 \mathrm{mM}, \mathrm{pH} 7.3$ ) was incubated for 10 minutes at room temperature $\left(25^{\circ} \mathrm{C}\right)$ with different procaine and $\mathrm{GH} 3$ concentrations: 0 (control), 0.5, 1.0, 2.0, 5.0, and $10 \mathrm{mM}$. After the addition of $50 \mu \mathrm{L}$ Amplex Red working solution $(300 \mu \mathrm{M})$, the samples were incubated for 10 minutes at room temperature and protected from light. The samples were diluted to $2000 \mu \mathrm{L}$ with PBS $1 x$ before the fluorescence measurements as above described. Results were also expressed as lipid peroxidation inhibition in percentage (\%).

2.6. Macrophage-Induced LDL Oxidation. The native LDL fraction was isolated from human fresh plasma using the density-gradient ultracentrifugation and prepared as described previously $[56,57]$. Human plasma was obtained from normolipidemic, apparently healthy volunteers selected by the Blood Donation Service of Burgundy/Franche-Comté, France. The human U937 monocyte-like cell line (European Collection of Cell Cultures, UK) was grown in the RPMI-1640 culture medium (Sigma-Aldrich, Product No. SLM-240-B) enriched with $10 \%$ heat-inactivated fetal calf serum and incubated for 48 hours with phorbol 12-myristate 13-acetate (Sigma-Aldrich, CAS No. 16561-29-8) for cell activation and 
differentiation into macrophages [58]. The adherent macrophages $\left(0.5 \times 10^{6}\right.$ cells $/ \mathrm{mL}$, in 6 -well plates) were further used for LDL oxidation experiments. The growth medium was replaced with the oxidation mixture containing serum-free Ham's Nutrient Mixture F-12 (Sigma-Aldrich, CAS No. 51651C) without phenol red, $8 \mu \mathrm{M} \mathrm{FeSO}_{4}$ (Sigma-Aldrich, CAS No. 7782-63-0), and pure LDL at a final concentration of $100 \mu \mathrm{g}$ protein $/ \mathrm{mL}$ [58]. The cells were exposed for 24 hours at $37^{\circ} \mathrm{C}$ in a $5 \% \mathrm{CO}_{2}$-humidified atmosphere to the oxidation medium supplemented in different procaine and $\mathrm{GH} 3$ concentrations: 0 (control), $0.5,1.0$, and $2.0 \mathrm{mM}$ procaine hydrochloride equivalents. Lipid peroxidation end-products generated by macrophages were estimated by thiobarbituric acid reactive substances (TBARS) measurements in the cell culture medium. After incubation, the cell oxidation medium was removed, and lipid peroxidation was stopped with $1 \mathrm{mM}$ EDTA (Sigma-Aldrich, CAS No. 60-00-4) and 0.2 mM butylhydroxytoluene (BHT, Sigma-Aldrich, CAS No. 25013-16-5) at $4^{\circ} \mathrm{C}$. Detached cells present in the medium mixture were discarded by centrifugation ( $207 \mathrm{x} g, 5 \mathrm{~min})$. The TBARS from the supernatant were determined according to [58] with trichloracetic (TCA, Sigma-Aldrich, CAS No. 76-03-9)/thiobarbituric (TBA, Sigma-Aldrich, CAS No. 504-17-6)/hydrochloric ( $\mathrm{HCl}$, Sigma-Aldrich, CAS No. 7647-01-0) acid $(13.6 / 0.36 / 2.4 \%, w / v)$ reagent mixture and measured at $532 \mathrm{~nm}$, using a Lambda Bio10 Perkin-Elmer spectrophotometer. TBARS were expressed as nmoles of malondialdehyde (MDA) using a calibration curve of 1,1,3,3-tetramethoxypropane (Sigma-Aldrich, CAS No. 102-52-3). Results were expressed as lipid peroxidation inhibition in percentage (\%), calculated using the following equation: $\left[\right.$ TBARS $_{\text {Control }}{ }^{-}$ TBARS $\left._{\text {Samples }}\right) /$ TBARS $\left._{\text {Control }}\right] \times 100$.

\section{Results}

3.1. Protective Effects of Procaine and GH3 on DNA Strand Breaks in Young versus Aged Individuals. DNA damage is known to be one of the mechanisms responsible for increasing mutagenesis risk, being involved in cellular survival, vascular aging, cancer, and neurodegenerative diseases [38, 59]. We hypothesized that procaine and $\mathrm{GH} 3$ protective effects would be more pronounced in older compared to young subjects. Treatment with GH3 (containing the same concentration of procaine) (Figures 1(a) and 1(b)) did not lead to any significant change in the amount of endogenous DNA strand breaks either in young or in aged individuals (two-way ANOVA $p>0.05$ ). Contrary, treatment with different procaine concentrations significantly reduced the amount of endogenous DNA strand breaks in a dose-dependent manner (two-way ANOVA $p=0.0002$ ) but independent of age. However, treatment with $1 \mathrm{mM}$ procaine decreased the amount of endogenous DNA strand breaks in aged (Friedman's $p=$ 0.0049; Dunn's multiple comparisons test $p=0.0015$ ) but not in young individuals (Friedman's $p=0.1116$ ) when compared to their own group nontreated controls (Figures 1(c) and 1(d)).

Reduced antioxidant capacity $[1,60]$ and decreased DNA repair [61-63] are associated with ageing. Thus, it is conceivable that IR may induce higher DNA damage in PBMCs from

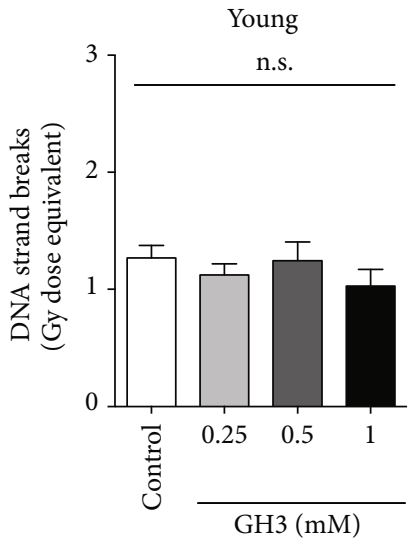

(a)

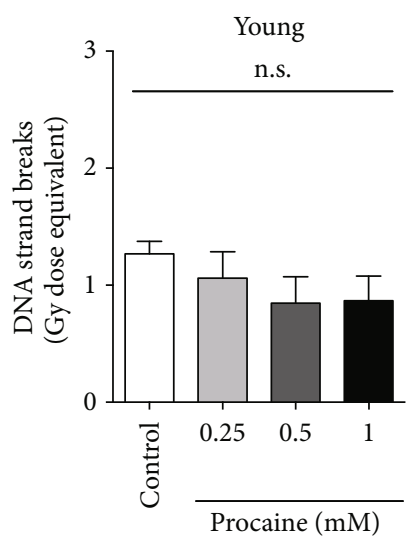

(c)

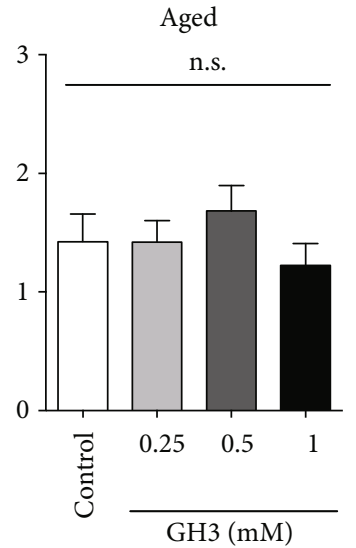

(b)

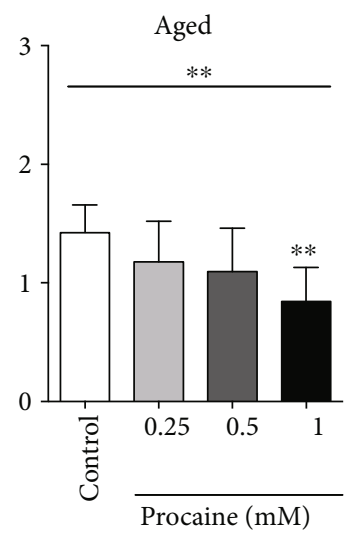

(d)
FIgURe 1: In vitro inhibitory effect of procaine (c), (d) and GH3 (a), (b) on DNA strand breaks in nonirradiated peripheral blood mononuclear cells (PBMCs) isolated from young (27 \pm 3 years) and aged ( $71 \pm 6$ years) individuals. Cells from young and aged subjects were divided in GH3- or procaine-treated or nontreated (control) groups, respectively. The cells were treated for 24 hours with $0,0.25,0.5$, or $1 \mathrm{mM}$ of procaine hydrochloride equivalents. Statistical analyses were performed using Friedman's test and Dunn's multiple comparisons test. Values, in Gy dose equivalent, represent means and SEM of 12 different individuals in each group. * Statistical significance when comparing against nontreated (control) samples, ${ }^{* *} p<0.01$; n.s.: nonsignificant.

elderly individuals than in those from young people. As expected, there was a significant (two-way ANOVA RM age $x$ dose interaction $p=0.0092$ ) effect of age on radiationinduced DNA strand breaks (Figure 2). All radiation doses $(2,4$, and $8 \mathrm{~Gy})$ induced a significantly higher number of DNA strand breaks in cells from aged individuals when compared with young individuals.

Pretreatment with GH3 before radiation significantly affected the radiation dose response (significant interaction between radiation and GH3 treatment; $p<0.0005$ ) in both young and aged individuals (Figures $3(\mathrm{a})$ and $3(\mathrm{~b})$ ). This effect was significant at radiation doses of 4 and $8 \mathrm{~Gy}$ $(p<0.005)$. Contrary, procaine (Figures 3(c) and 3(d)) did not affect the radiation dose response (no significant interaction between radiation and procaine treatment; $p>0.5$ ) in either young or aged subjects. 


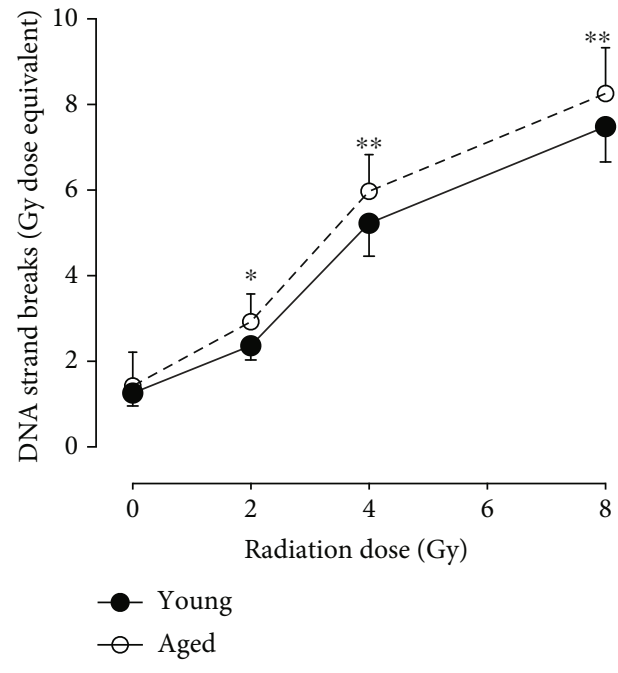

FIgURE 2: Endogenous and ex vivo radiation-induced DNA strand breaks in PBMCs isolated from young ( $27 \pm 3$ years, black circles) versus aged (71 \pm 6 years, white circles) individuals. Lines represent results from nonirradiated (radiation dose $=0 \mathrm{~Gy}$ ) and irradiated cells (radiation dose $=2,4$, or 8 Gy). Statistical analyses were performed using two-way RM ANOVA and Sidak's multiple comparisons test. Values represent means and standard deviations of 12 individuals in each group. * Statistical significance compared to young individuals, ${ }^{*} p=0.0366 ;{ }^{* *} p<0.01$.

\subsection{Protective Effects of Procaine and GH3 against Lipid} Peroxidation. In order to elucidate the distinct effects of procaine and GH3 on DNA damage, we evaluated the efficiency of different procaine and GH3 concentrations in preventing lipid peroxidation in various in vitro experimental models.

First of all, the antioxidant effects of procaine and GH3 were assessed in a human lymphoblastoid cell line. The purpose of this experiment was to evaluate the effects of procaine and GH3 using a cellular experimental model that mimics physiological targets to be protected in vivo against oxidative stress resulting from proinflammatory conditions (Figure 4). Procaine and GH3 similarly reduced the generation of cell membrane lipoperoxides at 5 and $10 \mathrm{mM}$ concentrations (Figures 4(b) and 4(c)). However, at the lowest concentration $(2.5 \mathrm{mM})$, their effects significantly differed $(p<0.0001)$, as $\mathrm{GH} 3$ was more effective in reducing the generation of membrane lipoperoxides, showing similar activity to curcumin (Figure 4(a)).

Both procaine and GH3 showed a significant $(p<0.0001)$ dose-dependent inhibitory effect on lipid peroxidation in human serum samples and in rat liver mitochondria. Interestedly, in human serum (Figure 4(d)), GH3 showed a significantly $(p<0.0001)$ higher lipid peroxidation inhibition compared to procaine, whereas in rat liver mitochondria (Figure $4(e))$, the inhibitory effect of procaine was significantly higher $(p<0.0001)$.

Further, the effect of procaine and GH3 on cell-mediated induced LDL oxidation was investigated. In this purpose, LDL lipoperoxides were generated by incubating macrophages differentiated from human monocytic U973 cells with human native LDL under prooxidant conditions. Endproducts of lipid peroxidation such as malondialdehyde
(MDA) and 4-hydroxynonenal (HNE) were measured as thiobarbituric acid reactive substances (TBARS). At all tested concentrations of procaine and GH3 significantly $(p<0.0001)$ inhibited TBARS formation. However, the inhibitory effect of GH3 was significantly $(p<0.0001)$ higher than that of procaine for the $2 \mathrm{mM}$ concentration (Figure $4(\mathrm{f})$ ).

\section{Discussion}

Ionizing radiation (IR) induces DNA damage and increases the risk of cancer [64]. Furthermore, the risk of radiationinduced carcinogenesis rises with age [10]. Therefore, taking age into consideration when investigating the effects of antioxidants, radioprotectors and scavengers on radiationinduced DNA damage, is of great interest in environmental, occupational, and medical research.

A significant fraction of DNA damage produced by IR is caused by free radicals generated during water radiolysis [65]. Since the repair capacity of ROS-induced DNA damage was reported to decrease with age [66], it is reasonable to hypothesize that radiation induces more cellular damage in older individuals. As mentioned before, radioprotective molecules may not only prevent the formation and facilitate the removal of free radicals but also reinforce natural antioxidant systems and enhance DNA repair. In order to allow cells to adjust their biochemistry in response to procaine and GH3, PBMCs were incubated for 24 hours. Furthermore, in order to avoid confounding effects due to immune stimulation through mitogens, we investigated the effect of procaine and GH3 in quiescent PBMCs known to be in G0 phase of the cell cycle [67]. Our results show that cells from older subjects were more susceptible to radiation. Whether or not these findings are in accordance with previously published data is difficult to assess. Early studies reported that the survival rate of lymphocytes from elderly individuals after ex vivo irradiation is approximately one-half from that of lymphocytes from young individuals [68]. Discordantly, a more recent study found an age-associated decrease in IRinduced apoptosis [69], and this finding was also confirmed in later studies $[70,71]$. Regarding radiation-induced DNA strand breaks, the number of $\gamma$-H2AX foci is higher in young than in old mice, which partially correlated with cellular proliferation and expression of DNA repair proteins [72]. However, in a human study including 172 individuals between 40 and 77 years of age, ex vivo irradiation of lymphocytes showed no significant differences in the induction of DNA strand breaks in aged versus young individuals [38]. Contrary, in another study including 31 individuals between 25 and 91 years old, an age-dependent increase in DNA single-strand breaks was observed in human lymphocytes immediately after ex vivo irradiation [73]. These discrepancies could be explained by the different experimental designs, cohorts, and/or methodologies. Further research is necessary in order to identify the factors that induce age-dependent radiosensitivity.

In this study, we did not detect any significant differences regarding the number of endogenous DNA strand breaks (without radiation) between young and elderly individuals. Our results showed that treatment of PBMCs with GH3 


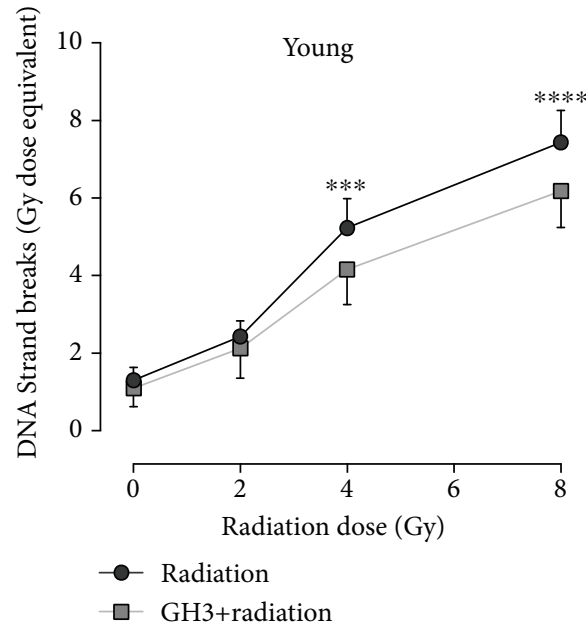

(a)

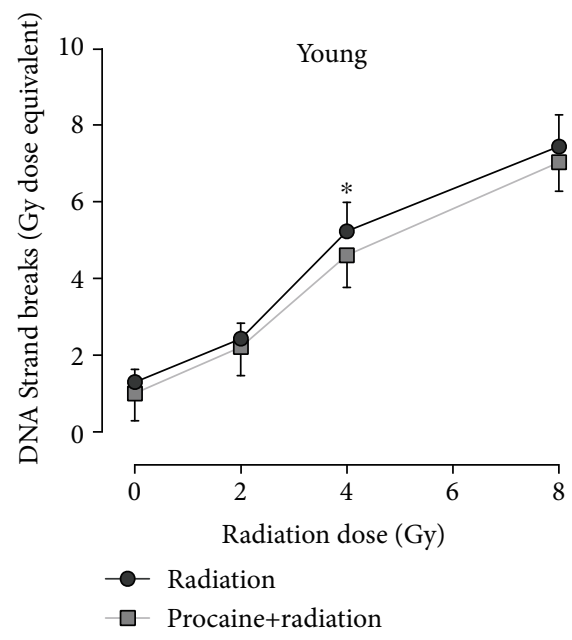

(c)

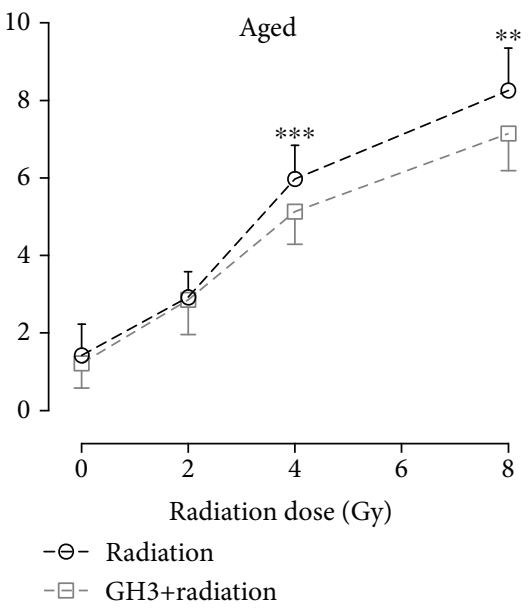

(b)

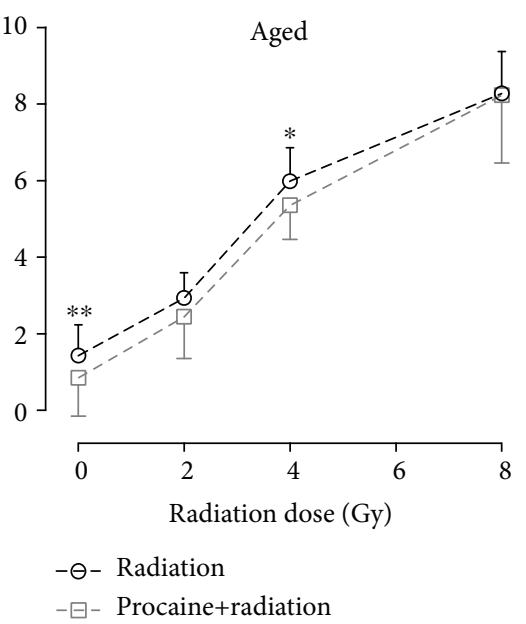

(d)

FIGURE 3: In vitro inhibitory effect of GH3 (1 mM) (a), (b) and procaine $(1 \mathrm{mM})$ (c), (d) on DNA strand break formation in irradiated peripheral blood mononuclear cells (PBMCs) isolated from young $(27 \pm 3$ years) and aged ( $71 \pm 6$ years) individuals. Cells were irradiated with $0,2,4$, or 8 Gy 24 hours after treatment with $1 \mathrm{mM}$ of procaine hydrochloride equivalents. Statistical analyses were performed using two-way RM ANOVA and Sidak's multiple comparisons test. Values represent means and standard deviations of 12 different individuals in each group. Statistical significance when comparing against nontreated (radiation) samples, ${ }^{*} p<0.05 ;{ }^{* *} p=0.0085 ;{ }^{* * *} p<0.0008$; **** $p<0.0001$.

reduced radiation-induced DNA strand breaks in both groups. However, it should be noted that the protective effect was slightly higher in young subjects, which could be explained by a higher interindividual variability in the aged group. Furthermore, the endogenous DNA strand breaks observed in nonirradiated cells could be reduced by procaine. There was a significant protective effect of $1 \mathrm{mM}$ procaine only within the elderly group probably due to the observed tendency of accumulating DNA strand breaks in aged individuals, but age did not have a significant effect on procaine dose response. Therefore, whether or not age influences the protective effect of procaine needs to be further investigated. These findings are intriguing: although the main active compound in GH3 is procaine, GH3 showed a protective effect against radiation, while procaine reduced the endogenous level of DNA strand breaks suggesting a slightly different mode of action.
Consequently, we aimed to evaluate whether procaine and GH3 differ in their antioxidant properties. In order to corroborate their antioxidant effects, we used novel, sensitive and specific, fluorescent in vitro methods, as well as various biological samples, relevant targets of oxidative damage such as lymphoblastoid cells, mitochondria, human serum, and oxidized LDL.

In our experiments comprising Jurkat cells, the lowest $\mathrm{GH} 3$ concentration showed similar effects to those induced by curcumin, while higher concentrations of procaine were needed to reach similar effects. Curcumin was used as a positive control of inhibition of lipid peroxidation, due to its well-known antioxidant action [50]. These results indicate that GH3 might have a higher capacity of preventing lipid peroxidation in a cellular system. Furthermore, in serumand mitochondria-based assays, the antioxidant capacity of procaine and GH3 increased in a dose-dependent manner. 


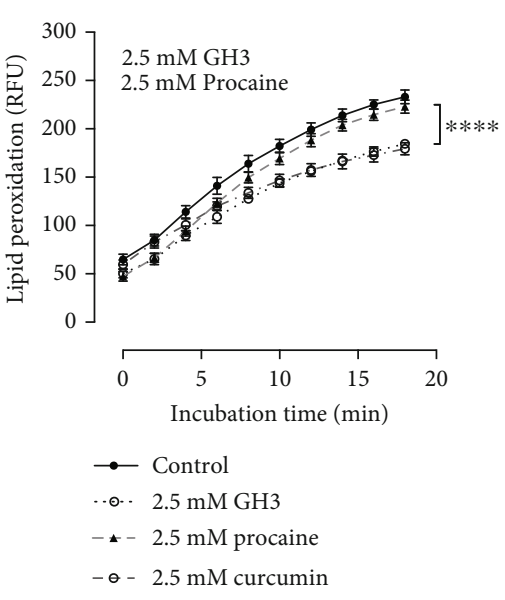

(a)

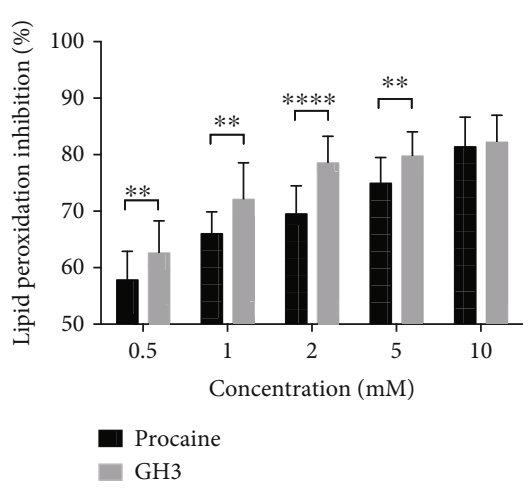

(d)

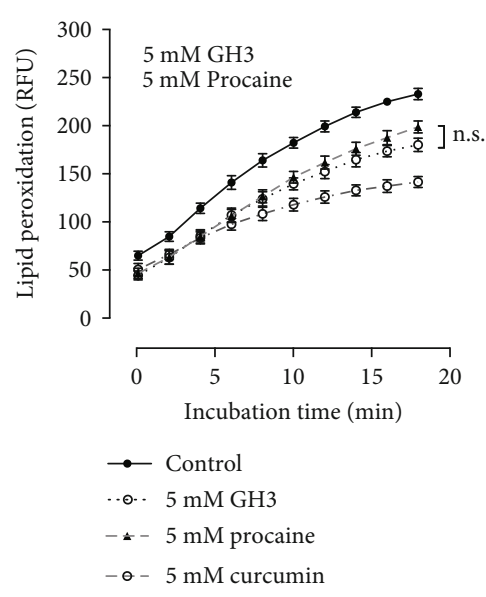

(b)

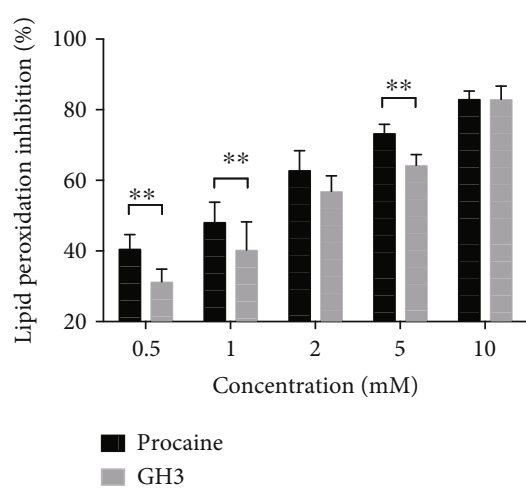

(e)

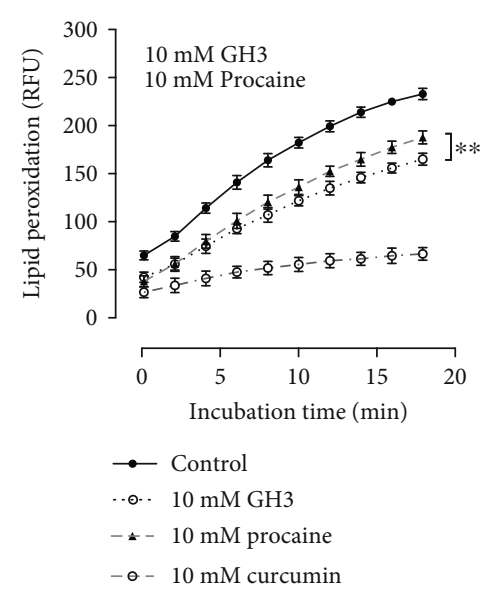

(c)

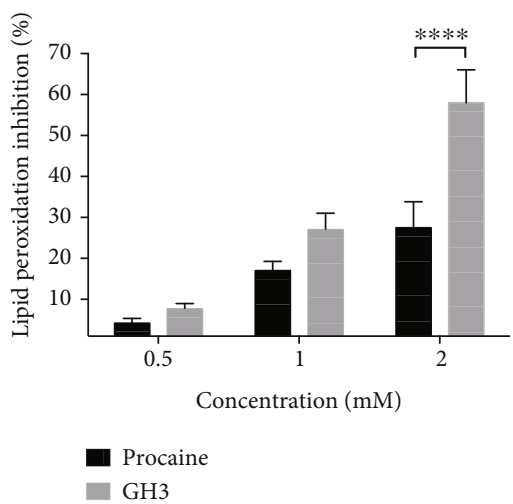

(f)

FIGURE 4: In vitro inhibitory effect of procaine and Gerovital H3 (GH3) on lipid peroxidation in Jurkat cells (a-c), human serum (d), rat liver mitochondria (e), and human macrophage-induced LDL oxidation (f). Jurkat cells were preincubated with 0 (control), 2.5 (a), 5.0 (b), or $10 \mathrm{mM}(\mathrm{c})$ of procaine hydrochloride equivalents for the indicated time points. Lipid peroxidation was induced by cumene hydroperoxide, and curcumin served as a control for inhibitory effect on lipid peroxidation. Human serum (d) and rat liver mitochondria (e) samples were pretreated with 0 (control), $0.5,1.0,2.0,5.0$, or $10 \mathrm{mM}$ of procaine hydrochloride equivalents. Human macrophage-induced LDL oxidation (f) was assessed in human U937 monocyte-like cell line. Cells were treated with 0 (control), 0.5, 1.0, or 2.0 mM of procaine hydrochloride equivalents, and TBARS was quantified. Statistical analyses for $(\mathrm{a}-\mathrm{c})$ and (f) were performed using two-way ordinary ANOVA and Sidak's multiple comparisons test. Statistical analyses for (d, e) were performed using two-way RM ANOVA and Sidak's multiple comparisons test. Error bars mean standard deviations of 3 or 4 experiments. Statistical significance between GH3 and procaine: ${ }^{* *} p<0.01 ;{ }^{* * *} p<0.0001$.

However, differences between the two compounds were also observed. While procaine was slightly more effective in protecting the mitochondrial membrane, GH3 was more efficient against serum lipoperoxidation. These outcomes could be explained through the different lipid or lipoprotein microenvironments present in these biological systems and/or through the different intrinsic antioxidant capacities or ROS scavenging actions of procaine and GH3, in counteracting or preventing lipid peroxidation [29-31]. Moreover, the reduced number of endogenous DNA strand breaks observed at 24 hours postexposure to procaine could be explained through inhibition of mitochondrial ROS production. Mitochondrial ROS also increases with age [74], which could explain the higher inhibition in aged individuals. In our macrophage-induced LDL oxidation model, GH3 showed a higher antioxidant effect than procaine, similar to the previously presented results for serum lipoproteins. These observations could provide additional information about the potential effect of procaine and $\mathrm{GH} 3$ regarding the oxLDL-macrophage interaction in the endothelial microenvironment [75].

Globally, we highlight the dose-dependent antioxidant effect of procaine and GH3; whereupon, GH3 seems to have a higher antioxidant capacity in serum and lipid- and lipoprotein-enriched biological samples compared to procaine. One could argue that GH3 also contains additional ingredients (potassium salts, benzoic acid-used as stabilizers and preservatives in the formulation of procaine hydrochloride) which could act as antioxidants. Indeed, in previous studies, using a nonenzymatic in vitro system for superoxide $\left(\mathrm{O}_{2}{ }^{-}\right)$generation, the antioxidant action of $\mathrm{GH} 3$ was attributed also to some GH3 of its components, [31, 76, 77]. However, for DNA damage experiments, cells were incubated 24 hours and thereafter centrifuged and suspended in isotonic 
buffer prior to radiation. Therefore, the GH3-mediated inhibition of radiation-induced DNA strand breaks cannot be explained by the presence of scavengers or antioxidants in cell culture medium; more likely, it is a matter of intracellular mechanisms, which could be attributed to procaine rather than to other additives in GH3. Furthermore, due to the higher procaine stability in $\mathrm{GH} 3$, the scavenger properties of procaine could be maintained for longer periods of time, explaining the GH3 protective effect on radiation-induced DNA strand breaks, in this case for both young and elderly individuals.

\section{Conclusion}

Due to the absence of recent and rigorous experimental studies involving procaine and $\mathrm{GH} 3$, we considered useful to explore the effects of procaine versus GH3 in the study of DNA damage and lipid peroxidation. In this work, we provide new evidence for differences in the antioxidant properties of procaine and GH3. Furthermore, procaine seems to affect the endogenous DNA strand breaks formation while $\mathrm{GH} 3$ prevents the radiation-induced DNA strand breaks. Age did not have any effect on $\mathrm{GH} 3$ treatment while procaine seems to have a slightly higher effect in the aged group although this remains to be confirmed by future extended and controlled studies. We conclude that both compounds, procaine, and GH3 have a different effect on DNA strand breaks formation which could be explained by their different antioxidative impact. Our findings are novel and constitute the basis for future studies.

\section{Data Availability}

The data used to support the findings of this study are available from the corresponding author upon request.

\section{Conflicts of Interest}

The authors declare that there is no conflict of interest regarding the publication of this paper.

\section{Authors' Contributions}

Maria Moreno-Villanueva and Daniela Gradinaru contributed equally to this work.

\section{Acknowledgments}

We wish to acknowledge the support consisting in the opportunity to make use of the equipment obtained under the framework of the EU-FP7 Project "MARK-AGE, European Study to Establish Biomarkers of Human Ageing" (grant agreement no. 200880). We also wish to thank Lotte Lenz and Katharina Schweinlin for their experimental support.

\section{Supplementary Materials}

Figure S1: effect of different procaine and GH3 concentrations on endogenous DNA strand break formation in PBMCs from three or two young subjects. Lines indicate an increase or decrease in the amount of DNA strand breaks after $24 \mathrm{~h}$ of ex vivo incubation when compared to their own initial ("physiological”) DNA strand breaks amount in each subject. (Supplementary Materials)

\section{References}

[1] D. Harman, "Aging: a theory based on free radical and radiation chemistry," Journal of Gerontology, vol. 11, no. 3, pp. 298-300, 1956.

[2] J. A. Reisz, N. Bansal, J. Qian, W. Zhao, and C. M. Furdui, "Effects of ionizing radiation on biological molecules-mechanisms of damage and emerging methods of detection," Antioxidants \& Redox Signaling, vol. 21, no. 2, pp. 260-292, 2014.

[3] A. Agrawal and R. K. Kale, "Radiation induced peroxidative damage: mechanism and significance," Indian Journal of Experimental Biology, vol. 39, no. 4, pp. 291-309, 2001.

[4] W. Luczaj and E. Skrzydlewska, "DNA damage caused by lipid peroxidation products," Cellular \& Molecular Biology Letters, vol. 8, no. 2, pp. 391-413, 2003.

[5] M. H. Yang and K. M. Schaich, "Factors affecting DNA damage caused by lipid hydroperoxides and aldehydes," Free Radical Biology \& Medicine, vol. 20, no. 2, pp. 225-236, 1996.

[6] K. Manda, A. Glasow, D. Paape, and G. Hildebrandt, "Effects of ionizing radiation on the immune system with special emphasis on the interaction of dendritic and T cells," Frontiers in Oncology, vol. 2, p. 102, 2012.

[7] J. Pajic, B. Rovcanin, D. Kekic, D. Jovicic, and A. P. S. Milovanovic, "The influence of redox status on interindividual variability in the response of human peripheral blood lymphocytes to ionizing radiation," International Journal of Radiation Biology, vol. 94, no. 6, pp. 569-575, 2018.

[8] J. Pajic, B. Rakic, B. Rovcanin et al., "Inter-individual variability in the response of human peripheral blood lymphocytes to ionizing radiation: comparison of the dicentric and micronucleus assays," Radiation and Environmental Biophysics, vol. 54, no. 3, pp. 317-325, 2015.

[9] A. Maguire, I. Vegacarrascal, L. White et al., "Analyses of ionizing radiation effects in vitro in peripheral blood lymphocytes with Raman spectroscopy," Radiation Research, vol. 183, no. 4, pp. 407-416, 2015.

[10] L. Hernández, M. Terradas, J. Camps, M. Martín, L. Tusell, and A. Genescà, "Aging and radiation: bad companions," Aging Cell, vol. 14, no. 2, pp. 153-161, 2015.

[11] B. Schuster, A. Ellmann, T. Mayo et al., "Rate of individuals with clearly increased radiosensitivity rise with age both in healthy individuals and in cancer patients," BMC Geriatrics, vol. 18, no. 1, p. 105, 2018.

[12] J. F. Weiss and M. R. Landauer, "Radioprotection by antioxidants," Annals of the New York Academy of Sciences, vol. 899, pp. 44-60, 2000.

[13] J. F. Weiss and M. R. Landauer, "Protection against ionizing radiation by antioxidant nutrients and phytochemicals," Toxicology, vol. 189, no. 1-2, pp. 1-20, 2003.

[14] A. Shirazi, E. Mihandoost, S. R. Mahdavi, and M. Mohseni, "Radio-protective role of antioxidant agents," Oncology Reviews, vol. 6, no. 2, article e16, 2012.

[15] T. A. Smith, D. R. Kirkpatrick, S. Smith et al., "Radioprotective agents to prevent cellular damage due to ionizing radiation," Journal of Translational Medicine, vol. 15, no. 1, p. 232, 2017. 
[16] D. Fusco, G. Colloca, M. R. Lo Monaco, and M. Cesari, "Effects of antioxidant supplementation on the aging process," Clinical Interventions in Aging, vol. 2, no. 3, pp. 377-387, 2007.

[17] H. Masaki, "Role of antioxidants in the skin: anti-aging effects," Journal of Dermatological Science, vol. 58, no. 2, pp. 85-90, 2010.

[18] I. Sadowska-Bartosz and G. Bartosz, "Effect of antioxidants supplementation on aging and longevity," Bio Med Research International, vol. 2014, pp. 1-7, 2014.

[19] S. Xavier, K. I. Yamada, A. M. Samuni et al., "Differential protection by nitroxides and hydroxylamines to radiationinduced and metal ion-catalyzed oxidative damage," Biochimica et Biophysica Acta, vol. 1573, no. 2, pp. 109-120, 2002.

[20] M. P. Blaustein and D. E. Goldman, "Competitive action of calcium and procaine on lobster axon. A study of the mechanism of action of certain local anesthetics," The Journal of General Physiology, vol. 49, no. 5, pp. 1043-1063, 1966.

[21] P. Seeman, "The Membrane Actions of Anesthetics and Tranquilizers, Pharmacological Reviews," in vol. 24, no. 4pp. 583655, The Williams and Wilkins Press, 1972.

[22] E. La Monaca and V. Fodale, "Effects of anesthetics on mitochondrial signaling and function," Current Drug Safety, vol. 7, no. 2, pp. 126-139, 2012.

[23] F. Bai, R. Michel, and P. Rossignol, "Effects of procaine on the oxidative phosphorylation of brain mitochondria from senescent rats," Mechanisms of Ageing and Development, vol. 26, no. 2-3, pp. 277-282, 1984.

[24] C. Tarba and C. Craciun, "A comparative study of the effects of procaine, lidocaine, tetracaine and dibucaine on the functions and ultrastructure of isolated rat liver mitochondria," Biochimica et Biophysica Acta, vol. 1019, no. 1, pp. 19-28, 1990.

[25] M. D. Mac Farlane, "Procaine HCl (Gerovital H3): a weak, reversible, fully competitive inhibitor of monoamine oxidase," Federation Proceedings, vol. 34, 1975no. 1, pp. 108-110, 1975.

[26] A. Villar-Garea, M. F. Fraga, J. Espada, and M. Esteller, "Procaine is a DNA-demethylating agent with growth-inhibitory effects in human cancer cells," Cancer Research, vol. 63, no. 16, pp. 4984-4989, 2003.

[27] T. Perls, "The reappearance of procaine hydrochloride (Gerovital H3) for antiaging," Journal of the American Geriatrics Society, vol. 61, no. 6, pp. 1024-1025, 2013.

[28] A. Ostfeld, C. M. Smith, and B. A. Stotsky, "The systemic use of procaine in the treatment of the elderly: a review," Journal of the American Geriatrics Society, vol. 25, no. 1, pp. 1-19, 1977.

[29] J. M. Lee, J. K. Suh, J. S. Jeong, S. Y. Cho, and D. W. Kim, "Antioxidant effect of lidocaine and procaine on reactive oxygen species-induced endothelial dysfunction in the rabbit abdominal aorta," Korean Journal of Anesthesiology, vol. 59, no. 2, pp. 104-110, 2010.

[30] D. Gradinaru, D. Margina, and C. Borsa, "In vitro studies regarding the antioxidant effects of procaine, Gerovital H3 and Aslavital," Revue Roumaine de Chimie, vol. 54, no. 9, pp. 761-766, 2009.

[31] C. Rusu and E. Lupeanu, "Inhibitory effect of procaine, Gerovital $\mathrm{H} 3$ and Aslavital on the production of superoxide radical," Romanian Journal of Gerontology and Geriatrics, vol. 10, no. 2, pp. 117-129, 1989.

[32] C. K. K. Nair and D. S. Pradhan, "Effect of procaine hydrochloride on DNA repair in Escherichia coli," Chemico-Biological Interactions, vol. 11, no. 3, pp. 173-178, 1975.
[33] M. Moreno-Villanueva, R. Pfeiffer, T. Sindlinger et al., "A modified and automated version of the 'Fluorimetric Detection of Alkaline DNA Unwinding' method to quantify formation and repair of DNA strand breaks," BMC Biotechnology, vol. 9, no. 1, p. 39, 2009.

[34] M. Moreno-Villanueva, T. Eltze, D. Dressler et al., "The automated FADU-assay, a potential high-throughput in vitro method for early screening of DNA breakage," ALTEX, vol. 28, no. 4, pp. 295-303, 2011.

[35] S. Brunner, D. Herndler-Brandstetter, C. R. Arnold et al., "Upregulation of mi R-24 is associated with a decreased DNA damage response upon etoposide treatment in highly differentiated CD8(+) T cells sensitizing them to apoptotic cell death," Aging Cell, vol. 11, no. 4, pp. 579-587, 2012.

[36] Z. Korwek, A. Bielak-Zmijewska, G. Mosieniak et al., "DNA damage-independent apoptosis induced by curcumin in normal resting human T cells and leukaemic Jurkat cells," Mutagenesis, vol. 28, no. 4, pp. 411-416, 2013.

[37] Z. Korwek, T. Sewastianik, A. Bielak-Zmijewska et al., "Inhibition of ATM blocks the etoposide-induced DNA damage response and apoptosis of resting human T cells," DNA Repair (Amst), vol. 11, no. 11, pp. 864-873, 2012.

[38] C. Garm, M. Moreno-Villanueva, A. Bürkle et al., "Genetic and environmental influence on DNA strand break repair: a twin study," Environmental and Molecular Mutagenesis, vol. 54, no. 6, pp. 414-420, 2013.

[39] S. Maynard, A. M. Hejl, T. S. T. Dinh et al., "Defective mitochondrial respiration, altered dNTP pools and reduced AP endonuclease 1 activity in peripheral blood mononuclear cells of Alzheimer's disease patients," Aging (Albany NY), vol. 7, no. 10, pp. 793-810, 2015.

[40] S. Maynard, G. Keijzers, Å. M. Hansen et al., “Associations of subjective vitality with DNA damage, cardiovascular risk factors and physical performance," Acta Physiologica (Oxford, England), vol. 213, no. 1, pp. 156-170, 2015.

[41] J. Morath, M. Moreno-Villanueva, G. Hamuni et al., "Effects of psychotherapy on DNA strand break accumulation originating from traumatic stress," Psychotherapy and Psychosomatics, vol. 83, no. 5, pp. 289-297, 2014.

[42] M. Moreno-Villanueva, A. Feiveson, S. Krieger et al., "Synergistic effects of weightlessness, isoproterenol, and radiation on DNA damage response and cytokine production in immune cells," International Journal of Molecular Sciences, vol. 19, no. 11, p. 3689, 2018.

[43] M. Moreno-Villanueva, G. von Scheven, A. Feiveson, A. Bürkle, $\mathrm{H}$. Wu, and N. Goel, "The degree of radiationinduced DNA strand breaks is altered by acute sleep deprivation and psychological stress and is associated with cognitive performance in humans," Sleep, vol. 41, no. 7, 2018.

[44] O. Alster, A. Bielak-Zmijewska, G. Mosieniak et al., "The role of nibrin in doxorubicin-induced apoptosis and cell senescence in Nijmegen breakage syndrome patients lymphocytes," PLoS One, vol. 9, no. 8, article e104964, 2014.

[45] F. Kalfalah, S. Seggewiß, R. Walter et al., "Structural chromosome abnormalities, increased DNA strand breaks and DNA strand break repair deficiency in dermal fibroblasts from old female human donors," Aging (Albany NY), vol. 7, no. 2, pp. 110-122, 2015.

[46] P. Palombo, M. Moreno-Villanueva, and A. Mangerich, "Day and night variations in the repair of ionizing-radiationinduced DNA damage in mouse splenocytes," DNA Repair (Amst), vol. 28, pp. 37-47, 2015. 
[47] M. Thomas, P. Palombo, T. Schuhmacher et al., "Impaired PARP activity in response to the beta-adrenergic receptor agonist isoproterenol," Toxicology In Vitro, vol. 50, pp. 29-39, 2018.

[48] M. Junk, J. Salzwedel, T. Sindlinger, A. Brkle, and M. MorenoVillanueva, "Mathematical modelling of the automated FADU assay for the quantification of DNA strand breaks and their repair in human peripheral mononuclear blood cells," $B M C$ Biophysics, vol. 7, no. 1, p. 9, 2014.

[49] D. Margina, D. Gradinaru, G. Manda, I. Neagoe, and M. Ilie, "Membranar effects exerted in vitro by polyphenols - quercetin, epigallocatechin gallate and curcumin - on HUVEC and Jurkat cells, relevant for diabetes mellitus," Food and Chemical Toxicology, vol. 61, pp. 86-93, 2013.

[50] D. Margină, O. Olaru, M. Ilie et al., “Assessment of the potential health benefits of certain total extracts from Vitis vinifera, Aesculus hyppocastanum and Curcuma longa," Experimental and Therapeutic Medicine, vol. 10, no. 5, pp. 1681-1688, 2015.

[51] A. Ungurianu, D. Margină, D. Grădinaru et al., "Lipoprotein redox status evaluation as a marker of cardiovascular disease risk in patients with inflammatory disease," Molecular Medicine Reports, vol. 15, no. 1, pp. 256-262, 2017.

[52] A. Ungurianu, O. Şeremet, E. Gagniuc et al., "Preclinical and clinical results regarding the effects of a plant-based antidiabetic formulation versus well established antidiabetic molecules," Pharmacological Research, vol. 150, p. 104522, 2019.

[53] A. Ungurianu, O. Șeremet, D. Grădinaru, C. Ionescu-Tîrgoviște, D. Margină, and R. Dănciulescu Miulescu, "Spectrophotometric versus spectrofluorometric assessment in the study of the relationships between lipid peroxidation and metabolic dysregulation," Chemical Biology \& Drug Design, vol. 93, no. 6, pp. 1026-1035, 2019.

[54] M. R. Wieckowski, C. Giorgi, M. Lebiedzinska, J. Duszynski, and P. Pinton, "Isolation of mitochondria-associated membranes and mitochondria from animal tissues and cells," Nature Protocols, vol. 4, no. 11, pp. 1582-1590, 2009.

[55] D. Gradinaru, D. Margina, C. Borsa et al., "Adiponectin: possible link between metabolic stress and oxidative stress in the elderly," Aging Clinical and Experimental Research, vol. 29, no. 4, pp. 621-629, 2017.

[56] L. Oziol, P. Faure, C. Vergely et al., "In vitro free radical scavenging capacity of thyroid hormones and structural analogues," The Journal of Endocrinology, vol. 170, no. 1, pp. 197-206, 2001.

[57] P. Faure, L. Oziol, Y. Artur, and P. Chomard, "Thyroid hormone (T3) and its acetic derivative (TA3) protect lowdensity lipoproteins from oxidation by different mechanisms," Biochimie, vol. 86, no. 6, pp. 411-418, 2004.

[58] L. Oziol, P. Faure, N. Bertrand, and P. Chomard, "Inhibition of in vitro macrophage-induced low density lipoprotein oxidation by thyroid compounds," The Journal of Endocrinology, vol. 177, no. 1, pp. 137-146, 2003.

[59] P. Bautista-Niño, E. Portilla-Fernandez, D. Vaughan, A. Danser, and A. Roks, "DNA damage: a main determinant of vascular aging," International Journal of Molecular Sciences, vol. 17, no. 5, p. 748, 2016.

[60] N. Muralidharan, T. Bhat, and N. S. Kumari, "A study on effect of ageing on the levels of total antioxidant and lipid peroxidation," International Journal of Contemporary Medical Research, vol. 4, no. 12, pp. 8-10, 2017.
[61] G. A. Garinis, G. T. J. van der Horst, J. Vijg, and J. H. J. Hoeijmakers, "DNA damage and ageing: new-age ideas for an age-old problem," Nature Cell Biology, vol. 10, no. 11, pp. 1241-1247, 2008.

[62] V. Gorbunova, A. Seluanov, Z. Mao, and C. Hine, "Changes in DNA repair during aging," Nucleic Acids Research, vol. 35, no. 22, pp. 7466-7474, 2007.

[63] A. A. Moskalev, M. V. Shaposhnikov, E. N. Plyusnina et al., "The role of DNA damage and repair in aging through the prism of Koch-like criteria," Ageing Research Reviews, vol. 12, no. 2, pp. 661-684, 2013.

[64] E. S. Gilbert, "Ionising radiation and cancer risks: what have we learned from epidemiology?," International Journal of Radiation Biology, vol. 85, no. 6, pp. 467-482, 2009.

[65] S. S. Wallace, "Enzymatic processing of radiation-induced free radical damage in DNA," Radiation Research, vol. 150, no. 5, pp. S60-S79, 1998.

[66] Y. A. Barnett and C. M. King, "An investigation of antioxidant status, DNA repair capacity and mutation as a function of age in humans," Mutation Research, vol. 338, no. 1-6, pp. 115-128, 1995.

[67] I. Yusuf and D. A. Fruman, "Regulation of quiescence in lymphocytes," Trends in Immunology, vol. 24, no. 7, pp. 380-386, 2003.

[68] R. Kutlaca, R. Seshadri, and A. A. Morley, "Effect of age on sensitivity of human lymphocytes to radiation. A brief note," Mechanisms of Ageing and Development, vol. 19, no. 2, pp. 97-101, 1982.

[69] K. Schnarr, I. Dayes, J. Sathya, and D. Boreham, "Individual radiosensitivity and its relevance to health physics," Dose Response, vol. 5, no. 4, pp. 333-348, 2007.

[70] A. Schmitz, J. Bayer, N. Déchamps, and G. Thomas, "Intrinsic susceptibility to radiation-induced apoptosis of human lymphocyte subpopulations," International Journal of Radiation Oncology • Biology • Physics, vol. 57, no. 3, pp. 769-778, 2003.

[71] M. A. Applebaum, A. D. Skol, E. E. Bond, M. Overholtzer, G. L. Bond, and K. Onel, "Radiation-induced apoptosis varies among individuals and is modified by sex and age," International Journal of Radiation Biology, vol. 90, no. 10, pp. 903-908, 2014.

[72] D. Hudson, I. Kovalchuk, I. Koturbash, B. Kolb, O. A. Martin, and O. Kovalchuk, "Induction and persistence of radiationinduced DNA damage is more pronounced in young animals than in old animals," Aging (Albany NY), vol. 3, no. 6, pp. 609-620, 2011.

[73] N. P. Singh, D. B. Danner, R. R. Tice, L. Brant, and E. L. Schneider, "DNA damage and repair with age in individual human lymphocytes," Mutation Research, vol. 237, no. 3-4, pp. 123-130, 1990.

[74] S. Kuka, Z. Tatarkova, P. Racay, J. Lehotsky, D. Dobrota, and P. Kaplan, "Effect of aging on formation of reactive oxygen species by mitochondria of rat heart," General Physiology and Biophysics, vol. 32, no. 3, pp. 415-420, 2013.

[75] D. Gradinaru, C. Borsa, C. Ionescu, and G. I. Prada, “Oxidized LDL and NO synthesis-biomarkers of endothelial dysfunction and ageing," Mechanisms of Ageing and Development, vol. 151, pp. 101-113, 2015.

[76] P. Gordon, J. Fudema, and A. Abrams, "Effects of Romanian and American procaine preparations on certain physiological aspects of ageing," Excerpta Medica, vol. 20, no. 7, p. 51, 1964.

[77] P. Lüth, “Aslan-Therapie mit Gero-H3 - Aslan," Zeitschrift für Allgemeinmedizin, vol. 60, no. 27, pp. 1162-1164, 1984. 